\title{
Morbidade referida e utilização de serviços de saúde em localidades urbanas brasileiras: metodologia*
}

\author{
Referred morbidity and the utilization of health services in urban \\ areas of south-eastern Brazil: methodology
}

\author{
Chester L. G. Cesar, Gerusa Maria Figueiredo, Márcia F. Westphal, Maria Regina A. \\ Cardoso, Maria Zilda de A. Costa e Vera Lucia Gattás \\ Departamento de Epidemiologia da Faculdade de Saúde Pública da U niversidade de São Paulo. São \\ Paulo, SP - Brasil (C.L.G.C.; M.F.W.; M.R.A.C.), Instituto de Saúde da Secretaria de Estado da Saúde \\ de São Paulo. São Paulo, SP - Brasil (G.M.F.; M.Z.A.C.; V.L.G.)
}

\begin{abstract}
Resumo
A reformulação do sistema de saúde, que vem ocorrendo em nível nacional e particularmente no Estado de São Paulo, tem motivado revisões do processo de planejamento, criando novas necessidades na área de informações. A criação do Sistema Único de Saúde e o processo de municipalização retomaram as propostas de integração das atividades curativas e preventivas, bem como a estruturação de sistemas de saúde regionalizados e hierarquizados. Nesse contexto, surgem como áreas de conhecimento, de particular interesse, o perfil de morbidade populacional e o padrão de utilização de serviços de saúde. As respostas a essas necessidades podem ser dadas por inquéritos domiciliares de saúde. Descreve-se a metodologia utilizada em um inquérito domiciliar realizado em municípios da região sudoeste da Área Metropolitana de São Paulo, SP, Brasil, no período de julho de 1989 a junho de 1990. Esse inquérito apresenta algumas características metodológicas específicas, entre elas o processo amostral utilizado, que definiu domínios para a amostra que permitiram análise de grupos pouco representados na população, como os menores de um ano de idade e a população idosa, bem como o ajuste da amostra a partir dos dados censitários de 1991 .
\end{abstract}

Serviços de saúde, utilização. Morbidade. Pesquisa sobre serviços de saúde, métodos.

\begin{abstract}
The reorganization of the Brazilian health system in the last few years has been based on the decentralization of the services and the integration of preventive and curative medicine, then creating new needs in term of the health information needed for health planning. The planning and administration of a
\end{abstract}

\footnotetext{
* Pesquisa financiada pela Secretaria de Estado da Saúde de São Paulo (Processo RUSP no 87.1.12539.1.3), pela Fundação de Amparo à Pesquisa do Estado de São Paulo (Processo n ${ }^{\circ}$ 91/1154-0) e pelo Conselho Nacional de Desenvolvimento Científico e Tecnológico/CNPq (Processo nº 501639/91-8). Correspondência para / Correspondence to: Chester L.G. Cesar - Departamento de Epidemilogia da Faculdade de Saúde Pública da Universidade de São Paulo. Av. Dr. Arnaldo, 715 - 01246-904 São Paulo, SP - Brasil. Fax: (011) 881.2108

Edição subvencionada pela FAPESP. Processo 95/2290-6.

Recebido em 26.5.1995. Aprovado em 21.12.1995.
} 
regionalized and integrated health system calls for information about the population's morbidity profile, and the description of the pattern of the utilization of the health services, which can be obtained by means of household health surveys. The methodology utilized in a household health survey carried out in the Metropolitan Area of S. Paulo, Brazil, from July 1989 to June 1990, is described. This survey has some distinct methodological characteristics, such as the sampling process that defined the domains by age and sex, allowing an analysis of populational groups less well represented in the general population, as that of less than one year of age and that of aged people, as well as the use of the results of the lastest Brazilian Census in 1991 for adjusting the sample.

Health services, utilization. Morbidity. Health services research, methods.

\section{INTRODUÇÃO}

Ao longo das últimas décadas, diferentes modelos de planejamento do setor saúde foram desenvolvidos, trabalhando em sua maioria com diagnóstico de saúde baseado em indicadores de mortalidade, dados sobre doenças de notificação compulsória, informações sobre produção dos serviços, bem como com a caracterização socioeconômica e ambiental da população.

Tais modelos deram conta, por muito tempo, da administração dos serviços públicos de saúde, na medida em que esses serviços restringiam suas atividades aos programas de prevenção e controle de algumas doenças específicas. Nesse contexto, a caracterização e o acompanhamento da população, utilizando-se das informações acima referidas, permitiam o planejamento e até certo ponto a avaliação das ações de saúde desenvolvidas.

Ao mesmo tempo, grande parcela do sistema de saúde não submetida ao controle direto do Estado, só se ajusta de forma indireta às suas linhas de trabalho. Nesse grupo encontra-se toda a rede hospitalar privada, bem como as outras organizações de prestação de serviços médicos privados. A este nível, o planejamento ocorre de uma forma indireta e está mais voltado a dar resposta à demanda que recorre a esses serviços, freqüientemente selecionada e assimilada de acordo com padrões de lucratividade. As informações aí trabalhadas dizem mais respeito ao controle das atividades desenvolvidas com finalidade administrativa (organização administrativa e financeira das instituições) e menos aos aspectos técnicos de prevenção e controle das doenças em nível populacional.

Na verdade, mesmo a rede hospitalar pública vem operando dissociada da rede básica de saúde, tendo como conseqüência uma organização voltada para o atendimento da demanda e, embora sem fins lucrativos, as informações aí trabalhadas dizem também respeito às atividades administrativas .

Nos últimos anos vem ocorrendo de uma forma mais concreta a integração dos serviços públicos de saúde, quer de nível federal, estadual ou municipal. Esse processo vem possibilitando algumas mudanças na estrutura e funcionamento dos mesmos, como maior integração do setor público e reformulações na articulação deste com o setor privado ${ }^{2,16,17}$.

Essa política de integração retoma de uma forma mais objetiva as questões da regionalização e hierarquização dos serviços de saúde, levando a uma redefinição do papel da rede básica com ampliação da sua capacidade resolutiva e sua definição como porta de entrada do sistema, que é visto sob a ótica sistêmica sem a dicotomia prevenção-cura.

Neste momento as questões referentes ao planejamento do setor saúde se ampliam. Dois fatos importantes mudam suas características.

Em primeiro lugar, o seu âmbito de ação deixa de ser, exclusiva ou primordialmente, a rede básica de serviços e passa a ser as atividades desenvolvidas por todo um sistema de saúde composto de instituições de diferentes complexidades e diferentes capacidades resolutivas (de unidades básicas às diferentes categorias de hospitais). Esse processo, obviamente, é mais centrado nas instituições públicas, embora venha também refletir-se no setor privado, a partir de novas formas de relacionamento deste com o setor público.

Em segundo lugar, a redefinição do papel da rede de saúde faz com que as Unidades Básicas de Saúde passem a ter uma capacidade resolutiva mais ampla, incorporando em maior grau atividades curativas e redefinindo o perfil da morbidade, objeto de seu controle, incluindo, por exemplo, programas de atenção às doenças crônicas. 
A estes dois fatos podemos acrescentar um terceiro, que também deve mudar as características do planejamento do setor saúde. Há um quase consenso na necessidade de descentralização administrativa e de operação dos serviços e na necessidade dos níveis regionais e locais participarem mais ativamente na definição das diretrizes de trabalho a serem implementadas por eles.

Esta ampliação do planejamento em saúde implica também redefinição das informações trabalhadas, incorporando às tradicionalmente utilizadas, aquelas referentes ao perfil de morbidade e padrões de uso de serviços. Há necessidade de se recorrer a novas fontes de informações, uma vez que as disponíveis, até então, são as referentes à demanda, o que não permite conhecimento do que vem ocorrendo em nível populacional. A resposta a estas novas necessidades é dada por levantamentos específicos ou implantação de novos sistemas de coletas de dados que possam periodicamente realimentar o sistema de saúde.

A revisão da literatura ${ }^{1,3,5,9,11}$ mostra a tendência crescente do uso de inquéritos populacionais para conhecimento da situação de saúde e do uso de serviços em nível populacional. Esses levantamentos iniciaram-se na década de 20, nos países industrializados, e a partir da década de 50, nos países em desenvolvimento. Eles vêm se caracterizando como levantamentos por entrevistas domiciliares para descrição da morbidade sentida pelas pessoas, do uso feito por elas dos diferentes serviços de saúde, preventivo e curativo, e como fonte de informações de formas "alternativas" de atenção à saúde.

Entre esses estudos vêm sendo privilegiados os de corte transversal único ou periódico, que utilizam entrevistadores leigos e levantam a morbidade sentida e expressa, sendo freqüentemente citados como "inquéritos domiciliares de saúde". Eles apresentam características próprias que os diferenciam de outros estudos de morbidade como os "inquéritos de saúde com exame clínico" que identificam morbidade definida por critérios clínicos e que utilizam em campo, pessoal especializado (freqüentemente médicos).

Kroeger ${ }^{10}$, em sua revisão dos métodos e resultados desses levantamentos, cita como principais propósitos dos inquéritos domiciliares de saúde: avaliar o estado de saúde da população; registrar o número de pessoas incapacitadas ou funcionalmente doentes; contribuir para a pesquisa no campo das doenças crônicas ou grupos específicos de doenças; ava- liar o uso de diferentes tipos de serviços de saúde; obter um "marco zero" para planejamento de saúde.

O mesmo autor aponta as seguintes vantagens desse modelo: a coleta de dados é relativamente rápida e fácil se comparada com sistemas permanentes de coleta; por outro lado, o custo é relativamente baixo quando comparado com "inquérito de saúde com exame clínico" ou "inquérito realizado através de diário"; outro ponto positivo é ser possível o uso de entrevistador não médico e a mensuração da saúde como é sentida pela comunidade. Além disso, estudos baseados na população possibilitam a classificação uniforme das informações referentes ao numerador e denominador, como também permitem agregar os dados de acordo com uma diversidade de variáveis explanatórias.

Projeto de pesquisa, financiado em grande parte pelo Programa Metropolitano de Saúde ${ }^{16}$, foi desenvolvido na região sudoeste da Área Metropolitana de São Paulo, formada por oito municípios*, onde estava implantado o Programa de Integração Docente-Assistencial (PIDA - Itapecerica da Serra) coordenado pela Faculdade de Saúde Pública da Universidade de São Paulo ${ }^{4}$. Os objetivos da pesquisa do PIDA foram auxiliar as instituições de saúde, da área, a conhecerem o perfil epidemiológico e o padrão de uso de serviços de saúde de suas populações, bem como criar um banco de dados que pudesse ser utilizado por alunos e pesquisadores.

Por estar centrada em um estudo de morbidade referida e de utilização de serviços de saúde a partir de entrevistas domiciliares, a presente pesquisa faz uso de metodologias pouco utilizadas em nosso meio, embora já largamente trabalhadas em outros países. Os resultados encontrados vêm permitindo uma análise crítica deste modelo de estudo na nossa realidade, identificando seu potencial, suas limitações e as dificuldades operacionais para sua realização.

O presente trabalho objetiva descrever a metodologia utilizada na realização do inquérito, desenvolvido como parte da implantação do Programa Metropolitano de Saúde na área estudada.

\section{METODOLOGIA}

O estudo desenvolveu-se em seis fases :

1. Revisão das questões teóricas e metodológicas;

2. Desenvolvimento do processo amostral;

3. Elaboração do questionário e realização do inquérito piloto;

\footnotetext{
* Taboão da Serra, Embu, Itapecerica da Serra, São Lourenço da Serra, Embu-Guaçu, Juquitiba, Cotia e Vargem Grande Paulista. Por ocasião da pesquisa São Lourenço da Serra era parte do Município de Itapecerica da Serra. Embora Vargem Grande Paulista já fosse um município autônomo, foi estudado conjuntamente com Cotia, pois os dois municípios possuíam um sistema de saúde integrado. Assim, foram estudadas seis "áreas" : Taboão da Serra, Embu, Itapecerica da Serra/São Lourenço da Serra, Embu-Guaçu, Juquitiba e Cotia/Vargem Grande Paulista.
} 

4. Análise do piloto, reformulação e elaboração final do projeto e do questionário;
5. Levantamento de campo (12 meses);
6. Análise dos dados e relatório final.

A primeira fase deu grande ênfase às questões metodológicas necessárias ao desenvolvimento do trabalho e que definiram as linhas gerais da pesquisa. Entre os aspectos metodológicos que merecem ser ressaltados estão os seguintes:

- Foram selecionados entrevistadores com segundo grau completo, sem treinamento prévio em saúde e que receberam um treinamento inicial para a realização das entrevistas. Sempre que possível esses entrevistadores foram selecionados entre residentes na área. A escolha de indivíduos sem treinamento prévio em saúde deveu-se à necessidade de registrar as informações prestadas exatamente como foram relatadas pelos entrevistados, evitando-se "traduzi-las" em linguagem técnica. Os entrevistadores foram acompanhados, avaliados e reciclados durante todo o período da pesquisa. Cerca de $10 \%$ da produção de cada um deles foi avaliada por nova entrevista parcial.

- As questões foram respondidas diretamente pelos adultos e pela mãe ou responsável por crianças menores de 14 anos, o que é preconizado por diversos autores para garantir maior confiabilidade das informações, principalmente aquelas referentes a problemas de saúde de menor gravidade.

- O período a que deveriam se referir as questões formuladas foi definido para obter o máximo de informações com a menor margem de erro possível, sendo um fator importante a memória do entrevistado. Assim, para as questões genéricas de morbidade utilizou-se o período de duas semanas anteriores à entrevista. Para o uso de medicamentos, questão de fácil esquecimento, três dias anteriores à entrevista. No caso da hospitalização, que é um evento marcante, o período a que se referem as entrevistas foi de doze meses, ocorrendo o mesmo para uso de serviços odontológicos. Doenças crônicas, alcoolismo e deficiências físicas foram estudadas a partir da sua prevalência no dia da entrevista. As entrevistas foram realizadas ao longo de doze meses, devido a possíveis variações sazonais das doenças ou a diferenças na utilização dos serviços de saúde ao longo do ano.

- O conhecimento da gravidade da doença é de grande importância, porém a sua mensuração não é tarefa fácil. Parece não haver um indicador ideal para esta medida, sendo mais utilizados critérios que apresentem em seu conjunto uma boa aproximação da realidade. Assim, para avaliar gravidade, foram incluídas questões referentes à limitação das atividades habituais e a falta ao emprego ou à escola.

- A caracterização do uso de serviços de saúde foi abordada a partir dos episódios de morbidade, sendo então investigada toda a seqüência de utilização ao mesmo tempo em que se procurou identificar os vários fatores que pudessem explicar o comportamento em relação a este uso. Foram analisados não só os serviços de saúde institucionais utilizados, como também identificado o uso de "serviços alternativos". Além disto, foram investigadas aquelas situações onde a procura não foi motivada por episódio de doença, mas por outras razões como exames preventivos e atestados de saúde.

A segunda fase do trabalho destinou-se ao desenvolvimento do processo amostral, que neste estudo apresentou algumas particularidades importantes:

- A primeira delas é que, para alcançar os objetivos propostos foi necessário trabalhar com uma amostra representativa da população residente em cada um dos municípios que compõem a região estudada. A técnica utilizada foi de "amostragem de área" com várias etapas de sorteio ${ }^{6,7,8}$.

Assim, em um primeiro momento, cada município foi considerado como um conjunto de setores ou subsetores censitários. Estes, em seguida, foram divididos em dois grupos chamados estratos. O critério utilizado para esta divisão foi o de densidade de domicílios, ou seja, o estrato 1 foi constituído por setores ou subsetores com grande quantidade de domicílios por $\mathrm{km}^{2}$, e o estrato 2, ao contrário, foi composto por setores ou subsetores com pequena concentração de domicílios*.

Após esta estratificação foram sorteados, em cada município, 30 setores ou subsetores, sendo que cerca de $70 \%$ destes pertenciam ao estrato 1 .

Os setores ou subsetores sorteados foram, em seguida, percorridos em toda sua extensão para que se pudesse atualizar os mapas (nome de ruas, aberturas de novas vias, desaparecimento de outras, etc.) e o número de domicílios em cada um deles. A seguir, para os setores ou subsetores de estrato 1 , houve uma segunda etapa de sorteio, onde foram selecionados 4 quarteirões do total de quarteirões do setor ou subsetor, e, para os setores ou subsetores do estrato 2, a segunda etapa de sorteio correspondeu à seleção de domicílios. Dessa forma, para o estrato 1 houve mais uma terceira etapa de sorteio onde foram selecionados os domicílios dentro dos quarteirões já sorteados.

Com este procedimento obteve-se uma amostra de domicílios representativa do total de domicílios de cada município da área.

\footnotetext{
* Para o cálculo da densidade de domicílios houve necessidade de obtenção da área de cada setor ou subsetor. Os mapas do IBGE que apresentam a delimitação dos setores, embora possuam escalas, não permitem o cálculo da área de cada um deles. A técnica utilizada para obtenção destas informação foi:

- a partir do mapa original em papel vegetal pertencente à FIBGE foram transcritos os setores censitários de cada município para outro papel vegetal e em seguida estes setores foram recortados um a um.

- estes papéis recortados que representavam os setores ou subsetores, foram secados em um dessecador e pesados em balança de precisão, sendo a sua massa comparada com a massa do recorte de um polígono regular (cuja área é facilmente determinada) feito do mesmo papel e obtida nas mesmas condições experimentais, obtendo-se assim a superfície dos setores e subsetores recortados no papel vegetal.

- com as informações das áreas e as escalas dos mapas obteve-se as áreas dos setores e subsetores em $\mathrm{km}^{2}$.
} 
- A segunda particularidade importante do processo amostral foi a definição dos grupos de idade e sexo a serem estudados, e para os quais deveriam ser garantidos números mínimos que permitissem análises posteriores. Estes grupos (domínios da amostra) foram: menores de 1 ano; 1 a 6 anos; 7 a 19 anos; 20 a 49 anos masculinos; 20 a 49 anos femininos; 50 anos e mais masculinos e 50 anos e mais femininos.

A definição desses domínios deveu-se principalmente ao fato de que alguns subgrupos populacionais, como os menores de 1 ano e os de idade muito avançada, representam uma proporção pequena do total da população e estes são os grupos que normalmente apresentam maiores prevalências de problemas de saúde e freqüentemente procuram mais os serviços de saúde. Assim, ao invés de entrevistar-se todos os moradores do domicílio, o que faria com que a amostra tivesse uma estrutura por sexo e idade semelhante à população, entrevistou-se números iguais de indivíduos para cada domínio, em cada município, o que fez com que a pirâmide populacional da amostra apresentasse uma configuração muito particular (Fig.1). O número de indivíduos a serem entrevistados em cada domínio foi definido como 200. Para o cálculo do tamanho da amostra utilizou-se da prevalência correspondente à situação de variabilidade máxima $(0,50)$, e admitiu-se como aceitável uma precisão tal que, em $95 \%$ das possíveis amostras, o erro máximo de amostragem (em valor absoluto) não ultrapassasse a $7 \%$. Esperando-se uma taxa de $80 \%$ de cobertura e resposta, foram sorteados, em cada domínio, 250 indivíduos para obter-se aproximadamente as 200 entrevistas.

— Uma terceira particularidade foi o processo de ponderação e ajuste. A utilização dos procedimentos anteriormente descritos, para a seleção da amostra, exige que antes de ser realizada a análise dos dados seja feita um ajuste. Este deve levar em conta o peso de cada domínio específico, na população total de cada um dos municípios, o que é feito normalmente com o inverso da "fração amostral" original. Além desse critério usual de pondera- ção optou-se por ajustar a amostra levando-se em conta também as informações do censo do IBGE para 1991, conforme método descrito por Holzer e col. ${ }^{6}$ e $\mathrm{Kish}^{8}$. Inicialmente foi estimada a população total de cada município para $1^{\circ}$ de janeiro de 1990 , a partir das populações censitárias de 1980 e 1991 . A distribuição por sexo e idade (domínios) desta população foi feita a partir da estrutura etária por sexo e idade em 1991. O peso específico para cada domínio foi obtido dividindo-se a população daquele domínio, obtida pelo censo de 1991, pelo número de indivíduos, do mesmo domínio, na população amostral. Este cálculo foi feito para todos os domínios em cada município, expandindo-se a população amostral de acordo com os pesos específicos encontrados.

A comparação entre a pirâmide populacional da área estudada, obtida a partir dos dados amostrais ponderados (Fig. 2), e a pirâmide populacional desta mesma área, definida a partir dos dados do censo de 1991 (Fig. 3), indica que a amostra ponderada de cada município representa no seu conjunto a população da área estudada.

Parte da terceira fase ocorreu simultaneamente com a segunda e correspondeu à elaboração do instrumento de coleta de dados. Nesta fase foram elaboradas as várias questões referentes aos itens levantados na entrevista domiciliar, bem como preparados os campos de codificação das questões. Terminado o endereçamento dos domicílios, foi retirada uma amostra de 408 indivíduos para um estudo piloto.

A realização e análise do estudo piloto, quarta fase, forneceu subsídios para a discussão final do tamanho da amostra e dos domínios inicialmente definidos. Além disso, este estudo forneceu elementos para a reformulação e simplificação do questionário.

A utilização do SPSS (Statistical Package for the Social Sciences) para análise dos dados levantados no estudo piloto permitiu que fossem feitas adequações nos campos de codificação e na estrutura geral do questionário, redefinindo os conjuntos e blocos de informações.

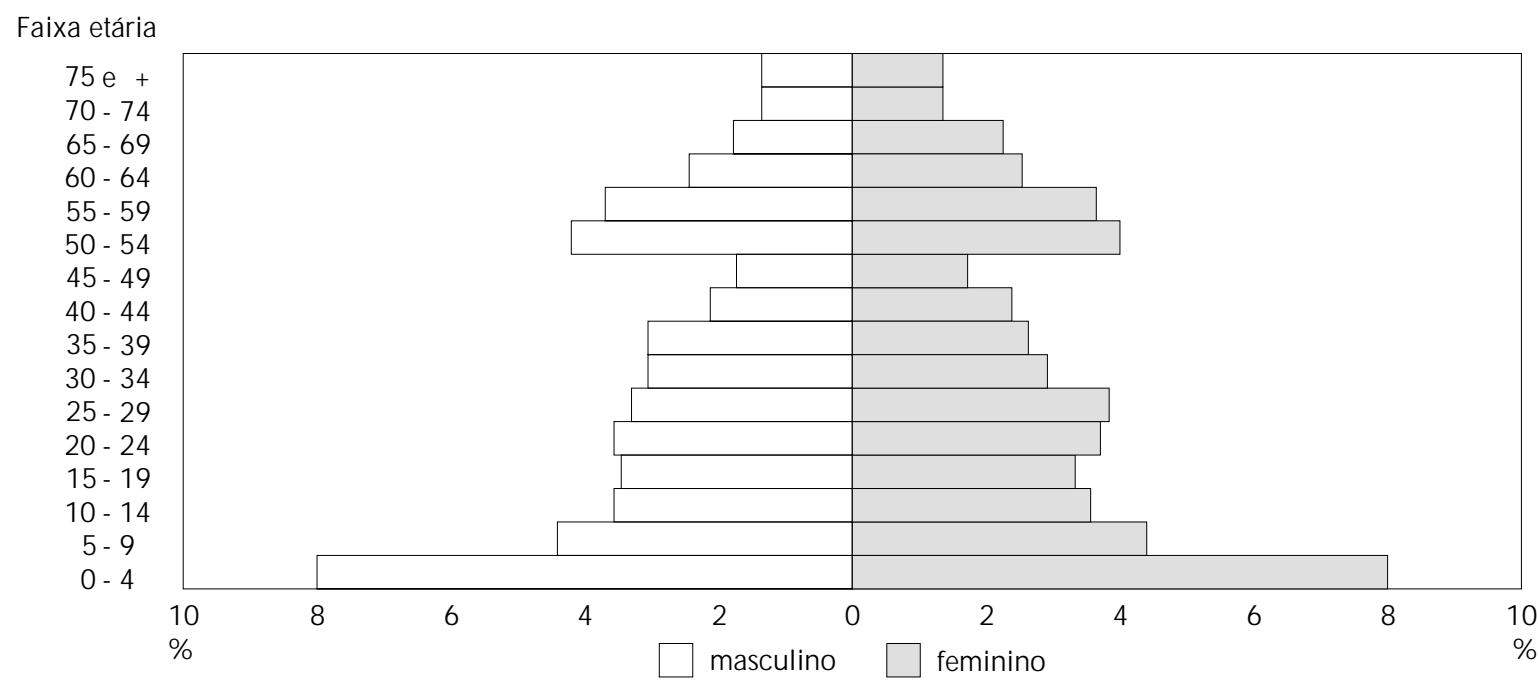

Figura 1 - Pirâmide populacional da amostra (dados não ponderados), região Sudoeste da Grande São Paulo, 1989-1990. 


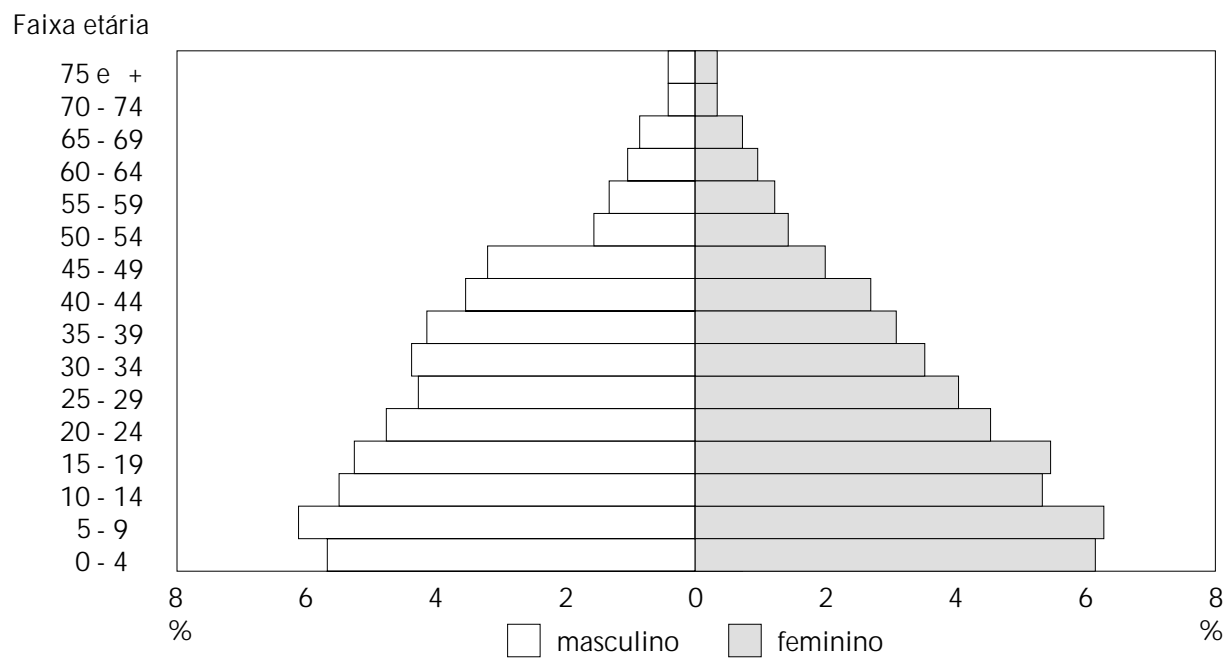

Figura 2 - Pirâmide populacional da amostra (dados ponderados), região Sudoeste da Grande São Paulo, 1989-1990.

O questionário aplicado contém os seguintes dados:

1. Identificação dos moradores do domicílio por família, com discriminação de sexo e faixa etária.

2. Características do domicílio segundo tipo de construção e condições de saneamento.

3. Para os indivíduos do domicílio sorteado:

No dia da entrevista; problemas crônicos e deficiências físicas.

Nas 2 semanas anteriores à entrevista; morbidade referida, utilização de serviços de saúde, encaminhamentos ambulatorial e hospitalar, realização de exames, avaliação do atendimento, gastos, fonte financiadora do atendimento recebido, e outros motivos de uso de serviços que não doenças.

Nos três dias anteriores à entrevista; uso de medicamentos.
Nos 12 meses anteriores à entrevista:

— hospitalização; causas, tempo de internação, avaliação do atendimento, gastos e fonte financiadora do atendimento recebido;

- serviço odontológico; causas, localização, avaliação do atendimento, gastos e fonte financiadora do atendimento recebido.

4. Para menores de 1 ano:

— utilização de serviços de saúde pela mãe durante a gestação, realização de pré-natal, condições de parto e puericultura, gastos e finaciamento do atendimento recebido.

5. Para menores de 5 anos:

- situação vacinal.

6. Para os maiores de 7 anos e os chefes de familia:

— condição socioeconômica.

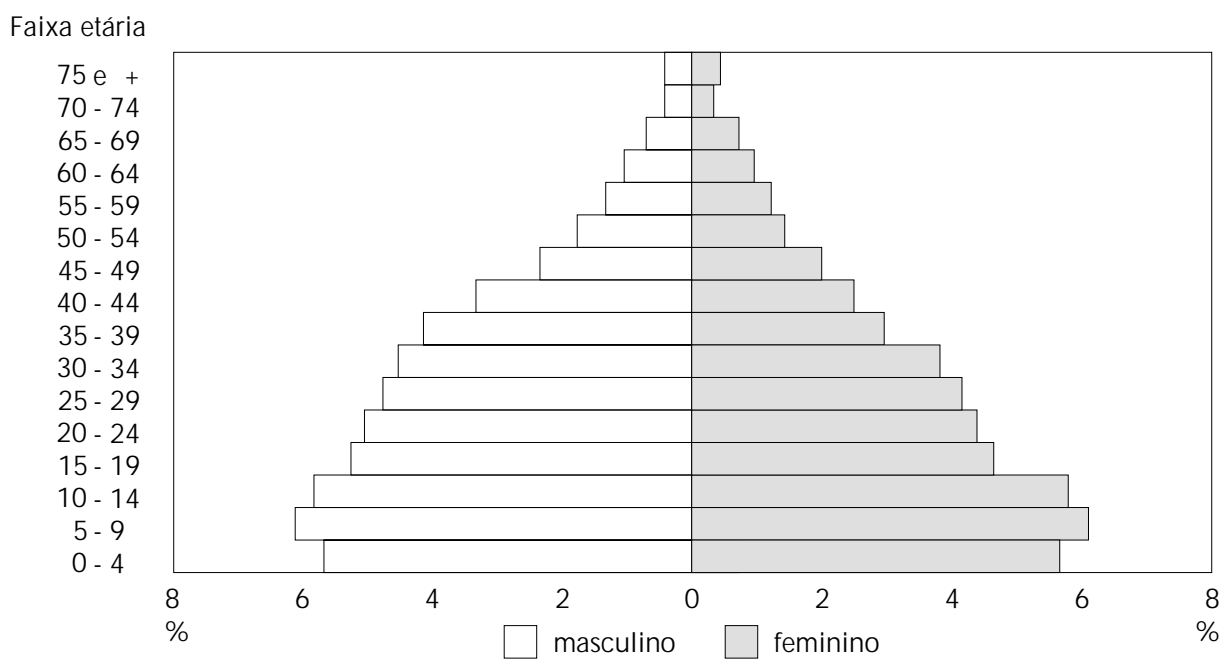

Fonte: FSEADE

Figura 3 - Pirâmide populacional da região Sudoeste da Grande São Paulo - Censo 1991. 
7. Para os maiores de 20 anos:

— alcoolismo através do teste $\mathrm{CAGE}^{13}$.

A grande maioria das questões do questionário foi fechada, com alternativas pré-definidas, porém a morbidade referida foi obtida através de questões abertas, sem a utilização de lista com perguntas específicas.

Antes do início do trabalho de campo houve divulgação da pesquisa através da assessoria de imprensa da Secretaria da Saúde, motivando uma boa cobertura sobre o estudo, o que pode em parte explicar a baixa frequiência de recusas.

A coleta de dados iniciou-se em julho de 1989 e prolongou-se até junho de 1990, correspondendo a quinta fase do trabalho. Durante esse período foram visitados domicílios distribuídos entre os oito municípios da área, e realizadas 10.199 entrevistas de um total de 10.796 indivíduos a serem entrevistados. Esta perda de $5,4 \%$ foi devida à recusa $(1,7 \%)$, ausência no domicílio $(2,4 \%)$ e outros motivos $(1,3 \%)$.

Durante a sexta fase foram executadas as seguintes atividades:

- Codificação das queixas referidas pelos entrevistados, utilizando-se a Classificação Internacional de Doenças - $\mathrm{CID}^{14}$. Esta tarefa foi desenvolvida por uma só pessoa com grande domínio e experiência na utilização da CID, com supervisão por profissional do Centro de Classificação de Doenças para Língua Portuguesa (OMS/FSP-USP).

- Codificação das questões referentes à condição socioeconômica; escolaridade, ocupação, renda e classe social. Foi feita não somente a codificação de cada questão individual como também permitiu que o conjunto delas definisse a "condição de classe social" do entrevistado. A análise destas questões e a identificação da inserção dos indivíduos nas diferentes frações de classe, a partir da inserção no processo produtivo, requereu um trabalho cuidadoso e um bom domínio dos conceitos relacionados à teoria de "classes sociais".

A utilização do conceito de classe social em estudos de saúde tem sido realizada como uma tentativa de ampliar o entendimento do processo saúde-doença na perspectiva da sua determinação social. Ainda que assunto controverso, optou-se por uma aproximação deste conceito através de sua operacionalização, utilizando-se como elemento referenciador o esquema proposto por Bronfman e Tuiram (citados por Lombardi ${ }^{12}$ ) no censo demográfico do México, e utilizado no Rio Grande do Sul ${ }^{12}$.

\section{REFERÊNCIAS BIBLIOGRÁFICAS}

1. CAMPOS, C.E.A. Os inquéritos de saúde sob a perspectiva do planejamento. Cad. Saúde Pública, 9 :190-200, 1993.

2. CAMPOS, G.W. de S. Um balanço do processo de municipalização dos serviços de saúde no Brasil. Saúde em Debate, 28:24-7, 1990
— Codificação do restante do questionário, composto em grande parte por "questões fechadas" com campos para codificação, utilizando-se um manual de codificação previamente elaborado.

\section{CONSIDERAÇÕES FINAIS}

O presente estudo requereu uma revisão dos trabalhos que fazem uma análise crítica das questões metodológicas dos estudos de morbidade e utilização de serviços de saúde ${ }^{10,15,18}$. Na elaboração e desenvolvimento da pesquisa foram observados os aspectos fundamentais que a literatura vem recomendando e que foram expostos na metodologia.

A análise do material levantado vem mostrando a importância deste tipo de inquérito com base populacional, pois ele permite uma melhor aproximação com a realidade de saúde da população, do que os estudos de demanda. As características do processo amostral e em particular os domínios definidos, possibilitaram análises da situação de saúde dos grupos menos representados na população, como os menores de um ano e a população idosa, que apresentam as maiores prevalências de problemas e que têm grande importância para o planejamento dos serviços de saúde. Este modelo de estudo permite ainda uma exploração mais aprofundada das diferentes variáveis que determinam o perfil de saúde e o padrão de uso dos serviços, como as variáveis socioeconômicas e em particular a "condição de classe social".

A utilização deste tipo de estudo requer uma avaliação prévia das características das informações necessárias, podendo-se optar por estudos de maior ou menor complexidade e com isto obtendo-se a melhor relação custo-benefício em cada situação concreta.

Os resultados preliminares desta pesquisa foram divulgados através de relatório técnico* encaminhado aos órgãos financiadores e aos serviços de saúde da área estudada, e análises posteriores do banco de dados vêm sendo apresentadas em congressos e encaminhadas como artigos para publicação.

3. CARVALHEIRO, J. da R. Levantamento das condições de saúde por entrevistas domiciliárias. Ribeirão Preto, 1975. [Tese de LivreDocência - Faculdade de Medicina de Ribeirão Preto da USP].

4. CARVALHO, L. de F. Integração docente assistencial: Projeto Itapecerica da Serra. Rev. Paul. Hosp., 34 (7/9):103-19,1986.

* CESAR, C. L. G. et. al. Morbidade referida e utilização de serviços de saúde no ERSA de Itapecerica da Serra, São Paulo - relatório final. Faculdade de Saúde Pública - USP. Secretaria de Estado da Saúde de São Paulo, 1992. 
5. CESAR, C.L.G. \& WALKER, G.J.A. Diversity in provision and utilization of maternal and child health care in an urban area of Brazil. Ann. Trop. Paediat., 6: 167-74, 1986.

6. HOLZER, C.E. et al. Sampling the household population. In: Eaton, W.W. \& Kessler, L.G., ed. Epidemiologic field methods in psychiatry. Orlando, Academic Press, 1985. p.23-48.

7. KALTON, G. Introduction to survey sampling. Berverly Hills, Sage University, 1983. (Paper Series on Quantitative Application in the Social Sciences no 7-35).

8. KISH, L. Survey sampling. New York, John Wiley, 1965.

9. KOHN, R. \& WHITE, K.L. ed. Health care: international study. Oxford, Oxford University Press, 1976.

10. KROEGER, A. Health interview in developing countries: a review of the methods and results. Int. J. Epidemiol., 12:465-81, 1983.

11. LEBRÃO, M.L. et al. Análise das condições de saúde e de vida da população urbana de Botucatu, São Paulo (Brasil). IV - Morbidade referida em entrevistas domiciliárias, 1983-1984. Rev. Saúde Pública, 25:453-60, 1991.
12. LOMBARDI, C. et al. Operacionalização do conceito de classe social em estudos epidemiológicos. Rev. Saúde Pública, 22:253-65, 1988.

13. MASUR, J. et al. Detecção precoce do alcoolismo em clínica médica através do questionário CAGE. J. Bras. Psiquiatr., 34:31-4, 1985.

14. ORGANIZAÇÃO MUNDIAL DA SAÚDE. Manual de classificação estatística internacional de doenças, lesões e causas de óbitos; $9^{\mathrm{a}}$ revisão, 1975. São Paulo, Centro Brasileiro de Classificação de Doenças da OMS, 1985. Classificação internacional de doenças (Revisão 1975). São Paulo, 1978.

15. ROSS, D.A. \& VAUGHAN, J.P. Health interview surveys in developing countries a methodologycal review with recomendations for future survey. London, School of Hygiene and Tropical Medicine, 1984. (EPS Publ. 4).

16. SECRETARIA DE ESTADO DA SAÚDE. Programa metropolitano de saúde. São Paulo, 1984.

17. TANAKA, O.Y. et al. Gerenciamento do setor saúde na década de 80, no Estado de São Paulo, Brasil. Rev. Saúde Pública, 26:185-94, 1992.

18. WHITE, K.L. Health surveys: who, why and what? World Health Statist. Quart., 38:2-14, 1985. 